\title{
THE OCEAN COLOUR RADIANCE VIRTUAL CONSTELLATION (OCR-VC)
}

\author{
James Yoder $^{(1)}$, Mark Dowell ${ }^{(2)}$, Nicolas Hoepffner ${ }^{(3)}$, Hiroshi Murakami ${ }^{(4)}$, Venetia Stuart ${ }^{(5)}$ \\ ${ }^{(1)}$ Chair, International Ocean Colour Coordinating Group, Woods Hole Oceanographic Institution, MS \#31, Woods \\ Hole, MA 02540 USA, Email: jyoder@whoi.edu \\ ${ }^{(2)}$ European Commission - Joint Research Centre, DG JRC Via E. Fermi, 2749, I-21027 Ispra (VA), Italy, \\ Email: mark.dowell@jrc.it \\ ${ }^{(3)}$ European Commission - Joint Research Centre, Global Environment Monitoring ,Unit I-21020 \\ Ispra (Va), Italy, Email: nicolas.hoepffner@jrc.it \\ (4) Japan Aerospace Exploration Agency (JAXA)/EORC, 2-1-1 Sengen, Tsukuba, Ibaraki 305-8505, Japan, \\ Email: murakami.hiroshi.eo@jaxa.jp \\ ${ }^{(5)}$ IOCCG (International Ocean Colour Coordinating Group) Executive Scientist, Bedford Institute of Oceanography. \\ P.O. Box 1006, Dartmouth, NS, CanadaB2Y 4A2, Email: vstuart@dal.ca
}

\begin{abstract}
The purpose of the OCR-VC (Ocean. Colour Radiance Virtual Constellation) is to ensure a long-term, sustained record of calibrated satellite ocean colour radiances (OCR). This manuscripts describes how an informal federation of space agencies and scientists will implement the activities required to accomplish this objective,
\end{abstract}

\section{PURPOSE OF THE OCR-VC AND INTRODUCTION}

The purpose of the OCR-VC (Ocean Colour Radiances-Virtual Constellation)is to ensure a longterm, sustained record of calibrated satellite ocean colour radiances (OCR) at key wavelength bands to determine the impact of ocean climate signals and climate change on ocean ecosystem and biogeochemical parameters. The strategy of the OCR$\mathrm{VC}$ is to produce calibrated OCR measurements from multiple satellites that can be merged together to improve global ocean spatial coverage and to produce time series for coastal and open ocean waters of scientific data products related to marine ecosystems and ocean biogeochemistry that can be sustained into the future. OCR data products currently in common use are phytoplankton chlorophyll-a (a measure of phytoplankton biomass), coloured dissolved organic matter, particulate carbon, suspended sediment and the diffuse attenuation coefficient (an indicator of water transparency). In addition, the direct retrieval of some inherent optical properties of the water enables the assessment of other essential elements of the marine system such as colored dissolved organic matter, particulate carbon, and suspended sediment.

The key space segment capabilities are the current and future polar-orbiting global OCR satellite missions (see Fig. 1). Of specific interest are SeaWiFS (Sea-viewing Wide Field of View Sensor), MERIS (Medium Resolution Imaging Spectrometer) on ENVISAT (Environmental Satellite), MODIS (Meridional
Overturning Circulation and Heat)-Aqua, OCM (Ocean Colour Monitor) on Oceansat-2, OLCI (Ocean Land Colour Instrument) on Sentinel $3 \mathrm{~A}$ and $3 \mathrm{~B}$, SGLI (Second-generation Global Imager) on GCOM-C (Global Change Observation Mission-Climate), VIIRS (Visible Infrared Imager Radiometer Suite) on NPOESS-C1 (National Polar-orbiting Operational Environmental Satellite System-Charlie 1), possibly VIIRS on NPP (NPOESS Preparatory Project), and future NASA (National Aeronautics and Space Administration) and CNES (Centre National d'Études Spatiales) instruments under consideration. Other instruments such as China's COCTS (Chinese Ocean Color and Temperature Scanner) on HY-1B (Ocean Color Satellite B) and Korea's GOCI (Geostationary Ocean Color Imager) on COMS-1 (Communication Ocean and Meteorology Satellite) are not collecting global data, and the OCR-VC can provide benchmarks (e.g. calibration, validation, and comparable algorithms) to these missions.

In addition to the space segment, projects such as the former NASA-supported SIMBIOS (Sensor Intercomparison for Marine Biological and Interdisciplinary Ocean Studies) project, the current ChloroGIN (Chlorophyll Global Integrated Network) (multiple sponsors), the ESA (European Space Agency)-supported GlobColour (European Service for Ocean Colour) and the European Commission/ESA/EUMETSAT (European Organisation for the Exploitation of Meteorological Satellites) -supported GMES (Global Monitoring for Environment and Security) Marine Core Service will be required to calibrate across sensors, validate data products and generate global and regional products from merged data sets.

\section{SCIENTIFIC AND OPERATIONAL RATIONALE FOR THE OCR-VC}

Satellite ocean colour imagery has many uses in oceanographic research, particularly in various studies of variability at meso-scale $(10-100 \mathrm{~km})$ to ocean basin 


\section{Ocean Colour Radiometry Missions}

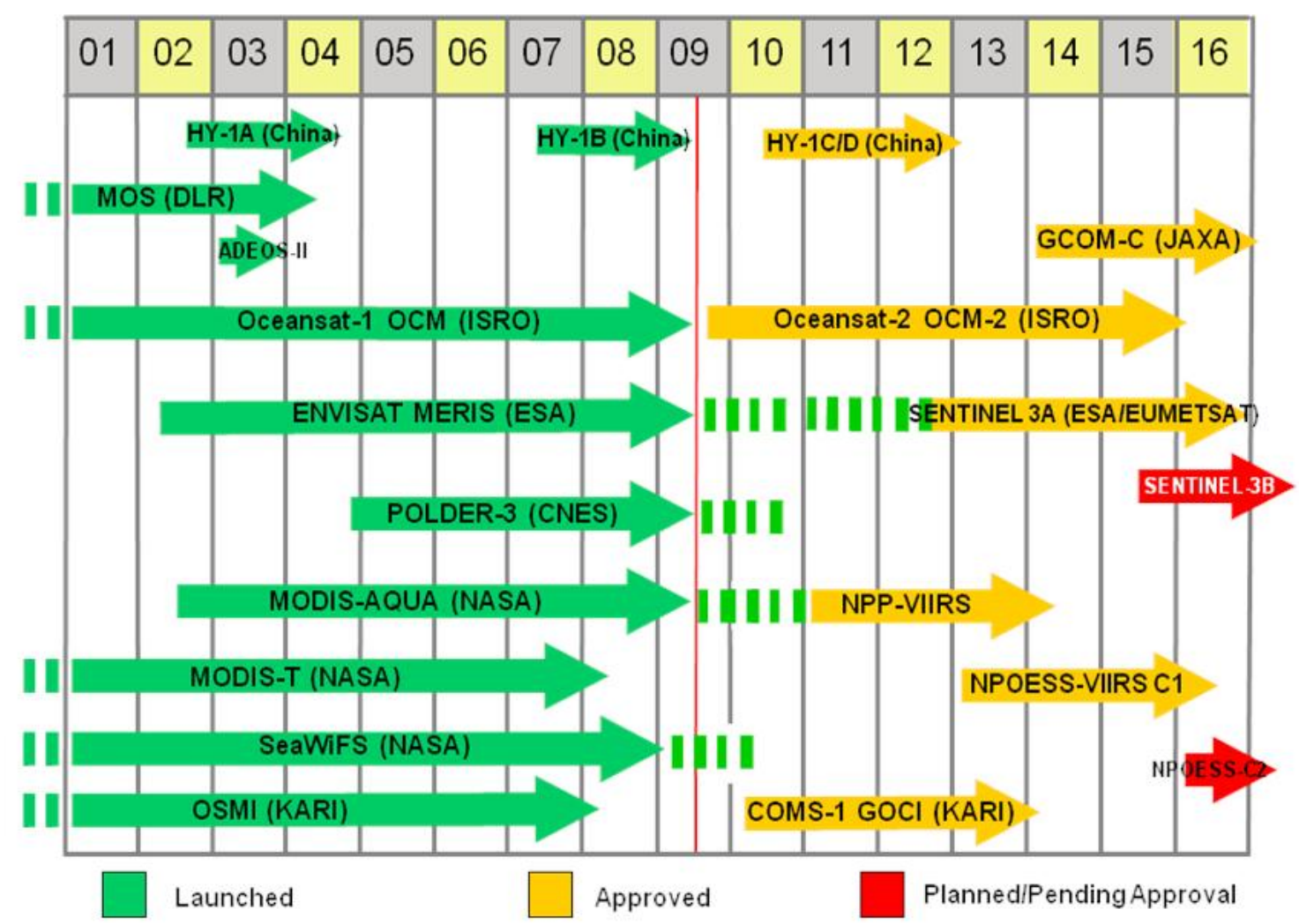

Figure 1. Ocean colour radiometry missions past, present and future. All are polar orbiters except for COMS-1 which will be on a geostationary platform to be inserted in orbit above the East China Sea.

spatial scales and time scales ranging from days to inter-annual [7]. It is the only observational tool that can make synoptic measurements of the global ocean related to ecological and biogeochemical processes. Global estimates of ocean primary production are now based on satellite OCR data. One of the main scientific contributions of the OCR-VC will be to sustain a long time series of global ocean colour measurements (which began in earnest with the launch of OCTS in 1996 and SeaWiFS in 1997 and with some early observations from CZCS (Coastal Zone Color Scanner) dating back to 1978) to help quantify inter-annual variability in the productivity of marine ecosystems and to separate the effects of inter-annual forcing (e.g. by ENSO (El Niño/Southern Oscillation)) from trends caused by changing climate. Four recent manuscripts [1], [2], [3] and [4] indicate that phytoplankton chlorophyll/carbon concentrations in large regions of the ocean are decreasing, possibly owing to climate change effects on ocean stratification. However, three of the four manuscripts are based on time series that began in fall, 1997, with the launch of
SeaWiFS. Thus, these time series were initiated during the beginning of one of the largest ENSO events of the century. Other studies [5], [6] and [7] showed that both the El Nino and La Nina phase of that ENSO event had significant impacts on satellite chlorophyll concentrations in many regions of the global ocean. The effects were evident in satellite OCR anomalies for several years, including high chlorophyll anomalies in the La Nina phase which began in 1998. Longer records will be required to sort out the effects of ENSO and other cyclical phenomena from trends related to a changing climate and changing ocean. The Global Climate Observing System (GCOS) requirements for OCR provide a concise summary statement as to the value of OCR data for studies of ocean climate and the potential impacts of climate change (GCOS-107, WMO/TD No. 1338, September 2006, in Sect. 3.2.4 ECV (Essential Climate Variables) Ocean Colour): "The primary benefits of OCR to GCOS are climate monitoring; chlorophyll-a linked to carbon-cycling including between the ocean and the atmosphere; and ocean particulate carbon estimated from ocean colour. 
Other applications include essential information for natural living-resource management and monitoring of the health of coastal seas."

In addition to its important contributions to ocean research and to impacts of ocean climate phenomena and change, products derived from satellite OCR also have operational uses as evidenced in GEO (Group on Earth Observations) requirements for ocean colour observations within the GEO Societal Benefit Areas (SBA) health, climate, ecosystems and agriculture as specified in the GEO 2009-2011 Work Plan (November 2008). The GEO 2009-2011 plan strongly supports the rapid development of virtual constellations including ocean colour radiometry (AR-09-02). The updated GEO plan points out the OCR-VC provides for "scientific data products related to marine ecosystems and ocean biogeochemistry for near-surface global ocean and coastal waters" and this simple statement summarizes the key data products that contribute to the tasks listed below from the GEO plan:

- Health. Task HE-09-01 (Information Systems for Health) includes a requirement for new observations characterizing atmospheric, soil, river and coastal pollution. Chlorophyll-a from satellite OCR has been used as an index of coastal eutrophication - a widespread type of coastal pollution caused by excess nutrient inputs. Harmful algal blooms (HABS) are also a form of coastal pollution and have been detected in OCR imagery.

- Task HE-09-02a (Aerosol Impacts on Health and Environment: Research, Monitoring and Prediction) has a goal of bringing all players interested in applying an integrated aerosol observational approach (including modeling) to reducing risks with respect to multiple hazards of aerosols. OCR imagery is used for large-scale aerosol mapping, including intercontinental movement of dust storms.

- Climate. Task CL-09-03a (Global Carbon Observation and Analysis System) is a requirement to support integrated global carbon observations. OCR products are used to estimate particulate and dissolved organic carbon in the ocean and along with SST for satellite-based calculations of $\mathrm{pCO} 2$ (Partial Pressure of Carbon Dioxide) in surface ocean waters.
- Ecosystems. Task EC-09-01a (Ecosystem Observation and Monitoring Network) requires the establishment of an ad hoc ecosystems classification task force, including ocean ecosystems, to create a robust and viable classification scheme for ecosystems. OCR is now being used for such a purpose, and the IOCCG (International Ocean Colour Coordinating Group) currently has an ocean ecosystem classification working group developing a report on how OCR is being used for ocean ecosystem classification.

- Task EC-09-01c (Regional networks for ecosystems). ANTARES (Advanced Neutron Tomography and Radiography Experimental System), which is cited as an example under this task, was founded by IOCCG and still has a close affiliation with IOCCG. It has been successfully extended into the global-scale ChloroGIN network. Workshops to strengthen observing capacity (for OCR) in developing countries have been an IOCCG activity for more than a decade with major workshops held in Southeast Asia, Africa, and South America.

- Agriculture Task AG-06-02 (Data Utilization in Aquaculture). This task mandates consulting with scientists and experts from the fisheries, aquaculture, coastal zone management and Earth observation communities at international and regional levels to identify opportunities for enhanced utilization of Earth observations in fisheries and aquaculture. See below for description of the SAFARI (Societal Applications in Fisheries \& Aquaculture using Remotely-Sensed Imagery) project.

\section{SUPPORTING PROGRAMS FOR THE OCR- $\mathrm{VC}$}

The OCR-VC will encourage cooperation among these programs and thus enlarge their contributions to scientific research and societal benefit areas.

\subsection{NASA's SIMBIOS Project}

SIMBIOS was based at the Goddard Space Flight Center (GSFC) from 1998-2003. Although funding for SIMBIOS ran out in 2003, many of the key products 
from this project are currently maintained at GSFC. In addition, the SIMBIOS Project and affiliated scientists published many reports and scientific manuscripts. SIMBIOS products of particular importance to the OCR-VC are: SeaWiFS Bio-optical Archive and Storage System (SeaBASS) which is an archive of very high quality bio-optical and other in situ measurements for validating satellite OCR products. Protocols for in situ measurements related to calibration and validation of satellite OCR products. Data base of sun photometer measurements which are important measurements for atmospheric correction of satellite radiances.

\subsection{SAFARI Project (Societal Applications in Fisheries \& Aquaculture using Remotely-Sensed Imagery)}

SAFARI was established in November 2007 as an element of GEO Task AG-06-02. The project, led by Trevor Platt (Bedford Institute of Oceanography, Canada), is funded by the Canadian Space Agency, with additional support provided by IOCCG, Bedford Institute of Oceanography and GEO. Remote sensing, in particular ocean-colour radiometry (OCR), is emerging as an important tool for fisheries research and management because of its high spatial and temporal resolution and low incremental cost. The primary objective of the SAFARI Projective is to accelerate the assimilation of Earth observations into fisheries research and ecosystem-based fisheries management on a world scale by facilitating the application of rapidly-evolving satellite technology to fisheries management questions.

\subsection{Chlorophyll Global Integrated Network (ChloroGIN)}

ChloroGIN is a combined POGO-GEO-GOOS (Partnership for Observation of the Global OceansGroup on Earth Observations- Global Ocean Observing System) initiative addressing GEO Task EC-06-07. ChloroGIN was established to develop a global network of in situ oceanographic measurements in conjunction with satellite-derived estimates, and to coordinate activities to strengthen observing capacity in developing countries. One of the key features of the network is that it is being developed using existing local capabilities. The core products provided include chlorophyll, sea surface temperature and light penetration. ChloroGIN was inspired by the ANTARES network of Latin America. Currently, there are four ChloroGIN regional centres (Latin America, southern Africa, Canada and Indian Ocean) that are linked by good communications to three northern centers (PML (Plymouth Marine Laboratory) in the UK, the European Commission, and USA). Each of these centers has its own set of priorities e.g. for
ChloroGIN-Africa the priorities include harmful algal blooms, while the Indian node of ChloroGIN uses satellite data for identifying potential fishing zones for the benefit of local fishermen. ChloroGIN is fulfilling a need to integrate in situ and remotely-sensed observations into a single network, and to work towards timely delivery of data and information for the benefit of society.

\subsection{ESA's GlobColour Project.}

The 3-year ESA funded GlobColour Project was established in November 2005 to develop a satellitebased, ocean-colour data set to support global carboncycle research, and to satisfy the scientific requirement for a long (10+ year) time-series of consistently calibrated global ocean-colour measurements with the best possible spatial coverage. This was achieved by merging data from the SeaWiFS, MODIS-Aqua and MERIS missions. The Full Product Set covers global daily merged, ocean-colour products for the time period 1997-2007, and is now freely available on the GlobColour website. At present the global daily delivery is in near-real-time, primarily to support operational oceanography. This service will continue well into the future by feeding into the EU GMES (European Union's Global Monitoring for Environment and Security) Marine Core Service that currently provides a suite of services to support Europe's decision makers. Furthermore, ESA's new CoastColour Project will develop and demonstrate MERIS Case 2 water retrievals over the world's coastal zones. Future availability of MERIS-quality ocean-colour data will be assured with the launch of the first Sentinel-3 satellite in 2012.

\section{STATUS OF THE OCR-VC}

The Strategic Implementation Team (SIT) of the Committee on Earth Observing Satellites (CEOS) approved the proposal for an OCR-VC in fall, 2008. The implementing organizations, primarily space agencies, affiliated with the IOCCG submitted the OCR-VC Implementation Plan to SIT in for consideration at their September, 2009 meeting. Participating agencies in the OCR-VC Implementation Plan include CNES, CSA (Canadian Space Agency), ESA, EUMETSAT, INPE (Instituto Nacional de Pequisas Espaciais), ISRO (Indian Space Research Centre), JAXA, JRC (Joint Research Centre), KORDI (Korea Ocean Research \& Development Institute), NASA, and NOAA (National Oceanic and Atmospheric Administration). Key components of the Implementation Plan are given below. The Implementation Plan was approved by CEOS (Committee on Earth Observing Satellites) in November, 2009. 


\section{OBJECTIVES OF THE OCR-VC IMPLEMENTATION PLAN.}

\subsection{Objective 1: Ensuring OCR continuity (Supporting GCOS requirement for an Ocean Colour ECV).}

- Activities to improve VIIRS, with launch of NPPVIIRS in 2011 and NPOESS C-1 no earlier than late 2012. NPOESS Program is currently trying to mitigate cross-talk issues with improved filter and better spectral out of band characterization for VIIRS on $\mathrm{C}-1$ platform. VIIRS Cal/Val program plan is now in the execution phase.

- GCOM-C and SGLI instrument is in development for launch in Q1 of 2014. Proposals responding to the first research announcement were submitted in Q1 2009, and science team, including international participation, was selected in Q3 of 2009. International collaboration for the mission is currently under discussion.

- OCM-2: ISRO's OCM-2 was successfully launched in September of 2009. ISRO will provide online access of Level 1-B to international research users at no cost. NASA will put OCM-2 data processing capability into SeaDAS.

- KORDI, CNES, NASA, NOAA, ISRO and others are evaluating geostationary or geosynchronous orbits for OCR sensors. KORDI will launch GOCI (first OCR sensor in geo orbit) in Q1 2010.

- IOCCG Working Group is evaluating user requirements as well as new capabilities for OCR measurements that will be provided by OCR sensors on geostationary/geosynchronous platforms.

- Pre-launch cooperative activities for Sentinel-3a and b (which carries OLCI - the MERIS follow-on instrument) are underway. Both the Sentinel-3A and 3B missions are now approved.

- Brazil and Argentina plan to launch a 2-sensor OCR mission with instruments for

- both global and regional coastal coverage. Mission specifications will be completed in Q4 of 2009.

5.2 Objective 2: Provide high quality data sets (Supporting GCOS requirement for an Ocean Colour ECV).

- Continue support for the MOBY (Marine Optical Buoy) bio-optical buoy which is used to provide high quality data for vicarious calibration of OCR sensors. All MOBY data is now available on-line for any user. MOBY-continuation (MOBY-C) will insure continuity of vicarious calibration across past, present and future ocean colour sensors. NOAA's goal is to get MOBY-C into the water before the end of 2011.
- Continue support for vicarious calibration activities using the BOUSSOLE (Buoy for the Acquisition of Long-term Time Series) buoy.

- NASA completed end to end reprocessing MODISTerra, MODIS-Aqua, SeaWiFS, OCTS and CZCS in Q4 2009.

- NASA sponsored an HPLC (High Performance Liquid Chromatography) round-robin experiment to improve quality of HPLC measurements of phytoplankton chlorophyll and other pigments for the SeaBAS archive and establish HPLC measurement protocols. Final report of the round-robin will be submitted Q3 of 2009.

- Continue interaction between NASA and ESA related to MERIS calibration and characterization and extend to pre-launch Sentinel-3 activities.

- MERIS will be reprocessed for third time by Dec 2009 using the same vicarious adjustment approach as used by NASA for SeaWiFS.

- MERMAID (MERis Matchup Insitu Database) is a centralized data base of in situ bio-optical data measurements concurrent with MERIS data extraction. The database currently contains data from sources such as AAOT (Venice tower), BOUSSOLE, and MOBY and shall support and sustain $\mathrm{Cal} / \mathrm{Val}$ activities including vicarious adjustment and algorithm development and validations. Further data are being acquired from a broader range of PIs through an invitation to contribute to this central tool in the MERIS validation strategy. MERMAID will be one of the tools for validating the next MERIS reprocessing.

- GEO Task EC-09-01c: Regional Networks for Ecosystems. ChloroGIN - promotes in situ measurement of chlorophyll in combination with satellite derived estimates. Approach is a network of regional networks with active networks in Canada, Africa, South America (ANTARES), Indian Ocean, and Europe. China expressed interest in establishing a network for Chinese regional waters.

- ESA's GlobColour project demonstrated the benefits of multiple sensor data merger as an important step towards an ocean colour Essential Climate Variable (ECV) for global products and has produced a 10-yr global data set based on MERIS, SeaWiFS and MODIS at $4.6 \mathrm{~km}$ resolution imagery. Spatial coverage is improved, e.g. what could be achieved in 8-days with SeaWiFS can be accomplished in 4-days by merging data from all 3 sensors. Data are freely available. Completed Q1 of 2009. GlobColour products will continue as part of the EC GMES Marine Core Service, i.e. MyOcean. 
- ESA is currently planning CoastColour for several coastal study areas using MERIS 300-m data. Proposals were due April 30, and Decision on "Champion Users" expected in Q4 2009.

- NASA to lead an IOCCG working group on Level-1 requirements for ocean colour sensors. Pre-launch and on-orbit requirements will be addressed, including vicarious calibration and on-orbit calibration.

\subsection{Objective 3: Data harmonization (Supporting GCOS requirement for an Ocean Colour ECV).}

- MERIS Quality Working Group (QWG) includes members of SeaWiFS and MODIS teams with the most recent meeting held in April 27-29, 2009 at ESRIN. QWG meetings will continue in the future.

- IOCCG WG is evaluating requirements and applications for bio-optical sensors on Argo floats for calibration and validation and to add vertical dimension for satellite OCR observations (WG

- Chair: Claustre, Laboratoire d'Océanographie de Villefranche). Development activities are underway and pilot studies are under consideration.

- IOCCG Working Group (WG) Geostationary OCR Sensors (WG Chair: D. Antoine, Laboratoire d'Océanographie de Villefranche). A specific question for the Geostationary WG is how can one relate the data from geostationary platforms for regional coverage (related to regional climate impacts as well as regional GEOSS (Global Earth Observation System of Systems) tasks) to polar orbiting instruments.

- IOCCG and OCR need better representation on Global Climate Observing System (GCOS) committees, as OCR is poorly represented now. For example, IOCCG should have a representative on the Ocean Observations Panel for Climate (OOPC). JRC will be organizing an interdisciplinary GCOS discussion workshop next year. The workshop will be cross-panel i.e. AOPC, TOPC and OOPC (Atmospheric Observation Panel for Climate, Terrestrial Observation Panel for Climate and Ocean Observations Panel for Climate) and will look at global biosphere production estimates and related ECVs (including of course OCR).

\subsection{Objective 4: Facilitate timely and easy access} to data (user interface)

- GEO Task AG-06-02: Data Utilization in Fisheries and Aquaculture. The Societal Applications in Fisheries and Aquaculture using Remotely-Sensed Imagery (SAFARI) project was created to accelerate the assimilation of Earth observation data into fisheries research and ecosystem-based fisheries management on a world scale. SAFARI is bringing together an international forum of leading experts to facilitate the application of rapidly-evolving satellite technology to fisheries management questions through collaboration and networking. This initiative, primarily funded by the Canadian Space Agency, is developed under the framework of the Group on Earth Observations. It also involves the participation of Canadian Department of Fisheries and Oceans and the IOCCG. SAFARI will support the OCR-VC by identifying opportunities for the enhanced utilization of Earth observations in fisheries and aquaculture, and by providing a framework for consulting with experts from fisheries, aquaculture, coastal zone management and Earth observation communities at regional and international levels. An IOCCG monograph on this topic will be printed by Q4 2009. SAFARI brochure was published in Q1 2009 and was translated into French. An international SAFARI symposium on remote sensing and fisheries will be held in Kochi, India, 15-17 February 2010. Proposal for a Phase 2 of SAFARI, to be combined with ChloroGIN, is under consideration. Decision expected Q1 2010.

- In response to an expression of interest articulated by Canadian Ocean stakeholders, the Canadian Space Agency (CSA) is upgrading ground infrastructure at the Canada Centre for Remote Sensing (CCRS) for the reception and processing of full resolution (300-m) MERIS data. The station mask covers most of the Canadian Artic, Pacific and Atlantic coasts. This initiative, established in collaboration with the European Space Agency and the support of the CCRS, will provide access to all recent MERIS Full Resolution (FR) Level 1 products (MER_FRS_1P) and Level 2 products (MER_FRS_2P) covering North America. The project started about 1 year ago with data currently processed in Europe. Beginning in Q4 2009, data will be processed in Canada rather than in Europe. Access is available to Canadian Govt. users or to ESA-approved users from any country.

- NOAA is developing an OceanWatch portal for VIIRS and possibly international sensors that will provide access to space-based observations of the global ocean for operations and climate applications.

- GEO Task WA-08-01g: Global Water Quality Monitoring. A GEO inland and coastal water quality remote sensing algorithm international workshop was held 19-21 May 2009 in D.C. (NASA supported). Report expected in Q4 2009. The workshop was related to GEO task WA-08-01g.

- INPE in cooperation with CSA will process MERIS FSR data for South American waters starting Q4, 2009 . 


\subsection{Objective 5: Capacity building and Outreach (GEO Task CB-09-03b: Establishing Regional Capacity Building Networks).}

- Training course on "Methods and Applications of Ocean Colour Remote Sensing in African Coastal and Regional Seas". Principle sponsors are JRC and Institute of Marine Sciences, University of Dar-esSalaam, Tanzania. It took place on 12 - 23 October 2009 at the University of Dar-es-Salaam, Stone Town, Zanzibar, Tanzania. Co-sponsors included IOCCG and other organizations.

- GEO Task ST-09-02: Promoting Awareness and Benefits of GEO. The organizers of the OceanObs'09 conference accepted a proposal from the OCR-VC leads for a white paper describing the OCR-VC. The conference occurred in Venice, Italy September 21-25, 2009, and is a major conference related to ocean observations and will have many attendees.

- GMES Africa will include remote sensing of marine and coastal areas. Implementation plan by early 2010 .

- The GKSS Research Centre, Geesthacht, Germany, in cooperation with the IOCCG, held an advanced training course/workshop for 15 participants on: Inversion Procedures in Ocean-Colour Remote Sensing: 10-14 August 2009, Hamburg Germany The course was organized by Dr. Roland Doerffer (GKSS) and dealt with complex waters with different optical components. The workshop to provided participants with an overview of inversion methods and models, prepared bio-optical models and training data sets for inversion methods, and taught participants how to use various inversion techniques.

\section{REFERENCES}

1. Antoine, D., A. Morel, H.R. Gordon, V.F. Banzon and R.H. Evans. 2005. Bridging ocean color observations of the 1980s and 2000s in search of long-term trends. J. Geophys. Res., 110, C06009. doi:10.1029/2004JC002620.

2. Behrenfeld, M.J., R.T.O'Malley, D.A. Siegel, C.R. McClain, J.L. Sarmmiento, G.C. Feldman, A.J. Milligan, P.G. Falkowski, R.M. Letelier and E. S. Boss. 2006. Climate-driven trends in contemporary ocean productivity. Nature 444, doi:10.1038/nature05317.

3. Gregg, W.W., N.W. Casey and C.R. McClain. 2005. Recent trends in global ocean chlorophyll, Geophys. Res. Lett. 32, L03606, doi:10.1029/2004GL021808.

4. Polovina, J.J., E.A. Howell, and M. Abecassis. 2008. Ocean's least productive waters are expanding. Geophys. Res. Lett., 35, L03618, doi:10.1029/2007GL031745,

5. Wilson, C. and D. Adamec. 2001. Correlations between surface chlorophyll and sea surface height in the tropical Pacific during the 1997-1999 El Nino -
Southern Oscillation event, J. Geophys. Res., 106: 31175-31188.

6. Yoder, J.A. and M.A. Kennelly. 2003. Seasonal and ENSO variability in global ocean phytoplankton chlorophyll derived from 4 years of SeaWiFS measurements. Global Biogeochem. Cycles, 17(4), 1112, doi:10.1029/2002GB001942, 2003.

7. Yoder, J.A. and M.A. Kennelly. 2006. What have we learned about ocean variability from satellite ocean color imagers? Oceanography 19: 152- 171. 